\title{
Dietary fatty acids mediate the secondary messenger phosphatidylinositol for microglial phagocytosis and migration
}

\author{
Smita Eknath Desale ${ }^{1,2}$ Subashchandrabose Chinnathambi ${ }^{1,2, *}$ \\ ${ }^{1}$ Neurobiology Group, Division of Biochemical Sciences, CSIR-National Chemical Laboratory, \\ Dr. Homi Bhabha Road, 411008 Pune, India \\ ${ }^{2}$ Academy of Scientific and Innovative Research (AcSIR), 411008 Pune, India
}

"To whom correspondence should be addressed: Prof. Subashchandrabose Chinnathambi, Neurobiology group, Division of Biochemical Sciences, CSIR-National Chemical Laboratory (CSIRNCL), Dr. Homi Bhabha Road, 411008 Pune, India, Telephone: +91-20-25902232, Fax. +91-2025902648. Email: $\underline{\text { s.chinnathambi@ncl.res.in }}$

\begin{abstract}
Alzheimer's disease is one of the neurodegenerative diseases, characterized by the accumulation of abnormal protein deposits, which disrupt the signal transduction in neurons and other glia cells. The pathological protein Tau and amyloid- $\beta$ contributes to the disrupted microglial signaling pathways, actin cytoskeleton, and cellular receptor expression. The important secondary messenger lipids i.e., phosphatidylinositols are largely affected by protein deposits of amyloid-beta in Alzheimer's disease. Phosphatidylinositols are the product of different phosphatidylinositol kinases and the state of phosphorylation at D3, D4, and D5 positions of inositol ring. PI 3, 4, 5-P3 involves in phagocytic cup formation and relates actin remodeling whereas PI 4, 5-P2-mediates the process of phagosomes formation and further fusion with early endosome. The necessary activation of actin-binding proteins such as Rac, WAVE complex, and ARP2/3 complex for the actin polymerization in the process of phagocytosis, migration is regulated and maintained by PI 3, 4, 5-P3 and PI 4, 5-P2. Dietary fatty acids depending on their ratio and types of intake influence secondary lipid messenger along with the cellular content of phaphatidylcholine and phosphatidylethanolamine. The deposited A $\beta$ deposits and extracellular Tau seed disrupt levels of phosphatidylinositol and actin cytoskeletal changes that hamper microglia signaling pathways in $\mathrm{AD}$. We hypothesize that being a lipid species intracellular levels of phosphatidylinositol would be regulated by dietary fatty acids. We keen to understand different types of phosphatidylinositol species levels in signaling events such as phagocytosis and actin remodeling owing to the exposure of various types of dietary fatty acids.
\end{abstract}




\section{Keywords}

Phosphatidylinositol, actin remodeling, phagocytosis, dietary fatty acids, Alzheimer's disease.

\section{Alzheimer's disease pathology}

Alzheimer's diseases being a neurodegenerative disorder indicates symptoms of cognitive decline, memory loss, and finally dementia over the advancing age. The extracellular senile plaques of amyloidbeta (A $\beta$ ) and intracellular neurofibrillary tangles of Tau (NFTs) are the hallmarks of the disease along with the neuroinflammation owing to activated glial network. The abnormal processing of A $\beta$ by $\beta$ secretase enzyme produce amyloid peptide of various lengths, $A \beta 40$, and $A \beta 42$ found to accumulate in the brain [1]. However different post-translational modifications of Tau protein detaches it from microtubule and triggers aggregation of Tau protein to produce intracellular NFTs. Tau protein released from the neuronal cells acts as a seed to introduce NFTs formation in neighboring neurons. Hence the Tau seed behaves like "prion" and is transmitted via synaptic or vesicular transportation [2]. Microglia on other hand intervene with the Tau propagation mechanism by mediating Tau secretion via exosomes [3, 4]. The activated microglia exacerbates Tau pathology by damaging dendrites and axons. With the recent studies, it is proven that Tau seed activates NLRP3-ASC inflammasome- a multi-protein complex that recruits pro-caspase-1 via ASC to cleave proinflammatory cytokine precursors and other signaling pathways involved in immune activation of microglia [5]. The Tau seed especially oligomers have been shown to modulate the actin cytoskeleton also contributes to the fact that extracellular Tau has a detrimental effect on various microglial signaling cascades [4]. Hence the presence of extracellular senile plaques of $A \beta$, NFTs, and Tau seed have a consequence on neuronal networks, signal transduction in neuro-glial cells and neuroinflammation. As comparison to $\mathrm{A} \beta$ oligomers, Tau aggregates and oligomers are also considered as neurotoxic in nature [6]. Due to the presence of excessive abnormal protein accumulation the process of glial activation leading to neuroinflammation. In this scenario, the hampered fundamental nature of glial cells to clear the pathoproteins contributes to neuroinflammation [7]. Production of cytokines, chemokines, and reactive oxygen species by immune cells of CNS and their duration decides the course of action or damage to the CNS. The activated microglia specifically alters transcriptional profile, produces cytokines, undergo actin rearrangement, which differentiates the pattern of receptors expressed on the cell surface. In the severe neuroinflammatory condition, the inflammatory response overpowers the repair mechanisms carried by microglia cells. $\mathrm{In} \mathrm{AD}$, the hyper-activation of microglia and elevated production of IL- $1 \beta$, TNF- $\alpha$, IL- 6 which contribute to neuronal synapse loss, A $\beta$ plaque deposition, and Tau hyperphosphorylation [8].

\section{A $\beta$ and Tau hampers Phosphatidylinositol signaling}


The amyloidogenic processing of amyloid precursor protein (APP) leads to the formation of insoluble monomer, dimer, oligomer, and aggregates of the amyloid peptide. The accumulation of amyloid plaques disrupts majorly the neurotransmission amongst the neuron, affects Tau pathology, and even contributes to excessive activation of glial cells. The insoluble amyloid oligomer found to be more neurotoxic to disrupt intracellular signaling [9]. A $\beta$ binds to various cellular receptors to induce neurotoxicity via mitochondrial dysfunction and oxidative stress leading to excessive calcium influx introduces toxicity [10]. The soluble $A \beta$ can interact with various receptors to activate downstream signaling pathways that produce reactive oxygen species, hyperphosphorylated Tau and induce inflammatory response in brain cells [1]. The phosphatidylinositols metabolism necessary for various intracellular signaling, which is affected by A $\beta$ oligomer by activating SHIP2 via Fc $\gamma$ RIIb receptor. The levels of important secondary messenger phosphatidylinositols have been disrupted that are involved in important cellular processes such as phagocytosis, migration, actin cytoskeleton remodeling. The A $\beta$ induced affected metabolism of phosphatidylinositols challenge Tau hyperphosphorylation by various protein kinases [11]. Amyloid- $\beta$ is also capable of disrupting the function of phosphatidylinositol-3 kinase (PI3K) an important enzyme in the conversion of Phosphatidylinositol 4, 5- diphosphate (PI 4, 5-P2) to phosphatidylinositol 3, 4, 5triphosphate and also involved in Akt-mTOR signaling pathway [12]. The soluble A $\beta$ oligomer also affects PI3K/Akt/GSK-3 $\beta$ pathway majorly causing neuronal death and Tau hyperphosphorylation [13]. The disrupted PI3K signaling targets Tau hyperphosphorylation, which impart pathological condition in AD. Aggregated form of A $\beta$ 25-35 significantly impair phosphatidylinositol related enzyme phospholipase $\mathrm{C}$ found in the brain during $\mathrm{AD}$ [14]. Apart from the involved enzymes in phosphatidylinositol signaling (PI), A $\beta$ directly reduced levels of PI 4, 5-P2 phospholipid that regulate various neuronal functions [15].

\section{Phosphatidylinositol influence actin remodeling}

The phosphorylated derivatives of phosphatidylinositols (PI) are the important secondary messenger in the cell. The phosphorylation at D3, D4, D5 positions of the inositol ring decides the type of response and the location of the derivative inside the cell. Phosphatidylinositol 4, 5- diphosphate (PI 4, 5-P2) and phosphatidylinositol 3, 4, 5- triphosphate (PI 3, 4, 5-P3) are the main lipid-derivative form in process of phagosome formation and early endosome maturation in phagocytosis [16]. The secondary lipid mediators PI 4, 5-P2 and PI 3, 4, 5-P3 concentrate at the plasma membrane to initiate the cellular processes such as endocytosis, phagosome maturation, actin polymerization and migration [17]. The PI 4, 5-P2 regulates actin dynamics by interacting with actin-binding proteins such as ARP2/3 complex, capping protein, WASP family proteins, and other actin-binding proteins [18]. Along with activating actin-binding proteins, PI 3, 4, 5-P3 specifically regulates membrane ruffling via protein kinase A (PKA) 
activity (Figure 1). PKA inhibition triggers a marked decrease in bulk accumulation of PI 3, 4, 5-P3 at membrane ruffles independent of Rac activation [19]. According to studies, the local synthesis of PI 4, 5P2 specifically via PIPK $\alpha$, which induces actin polymerization via ARP2/3 and increase local levels of PI 3, 4, 5-P3 for actin remodeling related to membrane ruffling [20]. After ruffling for endocytosis and phagosome formation the concentration kinetics of PI 4, 5-P2, and PI 3, 4, 5-P3 mechanistically linked to related actin remodeling. PI 3, 4, 5-P3 concentration sharply increases at the site of phagosomal cup formation and disappears once the phagosome has been sealed off from the plasma membrane. Whereas PI 4, 5-P2 levels significantly increased for circular ruffle formation subsequently decrease during endocytosis of foreign particle. The difference in levels of PI 4, 5-P2, and PI 3,4,5-P3 regulated by PI3K is mechanically important for actin remodeling and macropinosome formation $[16,21]$. The negatively charged lipid such as PI 3, 4, 5-P3 activates N-WASP, cdc42, which triggers ARP2/3-mediated F-actin polymerization for the podosomes formation. PI 3, 4, 5-P3 enriches membrane-associated actin regulation factors-1e (Myo1e) which links PI signaling to phagosome assembly [22]. The production PI 4, 5-P2 by the enzyme Phosphatidylinositol-5 kinase (PI5K) from PI 4-P is triggered at the cell membrane and overexpression of PI5K and reduced expression of phosphatase increase the levels of PI 4, 5-P2 important for rocketing of vesicles [23]. On the other hand the actin regulating protein binds PI 4, 5-P2 with a basic and hydrophobic amino acid. The interacting proteins include WASP superfamily protein, ARP2/3 complex, gelsoline family protein, and capping protein, which are also affected by surface density of PI 4 , 5-P2. PI 4, 5-P2 levels in cell manage F-actin levels along with their association with actin polymerization while the levels of PI 4,5- P2 depends upon the regulation of enzymes required for the production [24]. However the pool of PI 4, 5-P2 in cells is largely affected by extracellular stimulus [18]. In $\mathrm{AD}$, presence of extracellular $\mathrm{A} \beta$ oligomers decrease the levels of PI 4, 5-P2 and increases PI 3, 4, -P2 levels via SHIP-2 and causes hyperphosphorylation of Tau. The disrupted metabolism of PI due to A $\beta$ affects function of actin cytoskeleton and neurotoxicity, which contributes to neurodegeneration, synaptic failure in $\mathrm{AD}$. The maintenance of the metabolism of PI has become one of the therapeutic strategies for $\mathrm{AD}$ [11]. Tau is another important protein in $\mathrm{AD}$ apart from $\mathrm{A} \beta$, the hyperphosphorylation of Tau carried by PI3K pathway including GSK-3 $\beta$ [25]. The A $\beta$-induced increase in PI 3, 4-P2 levels trigger Tau hyperphosphorylation in neurons. In addition, disruption in PTEN eventually increases Tau hyperphosphorylation along with decreasing PI 4,5-P2 levels [11]. The A $\beta$ has induced the increased phospho-Tau intermediate in disease pathology and contributes to extracellular Tau seed.

\section{Phosphatidylinositol 4, 5-bisphosphate a regulator of actin remodeling}


In the initial studies, Roberto J. Botelho et al indicated the importance of PI 4, 5-P2 as the lipid mediator which can cross the membrane and regulate the transient remodeling of actin filaments at the site of

\section{Figure 1}

\section{Intracellular signaling of phosphatidylinositol in actin remodeling and phagocytosis}

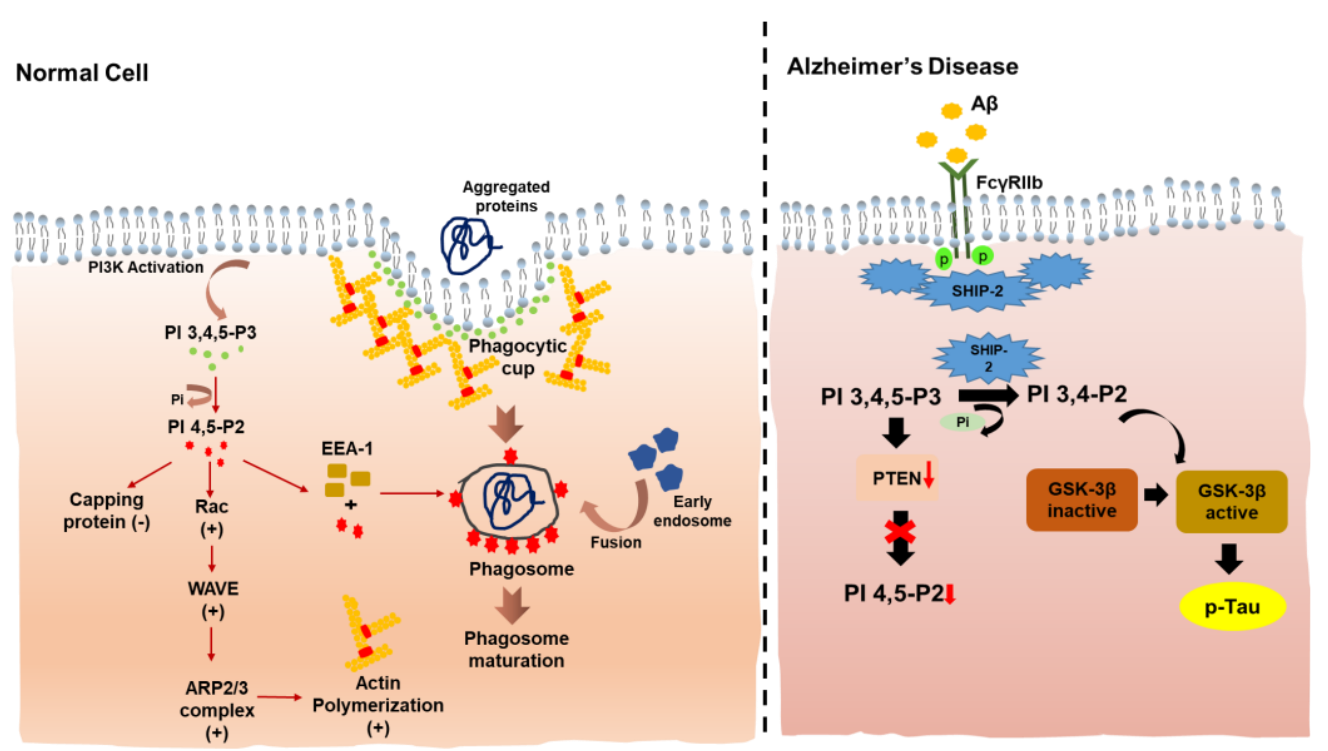

Figure 1. Intracellular signaling of phosphatidylinositol in actin remodeling and phagocytosis. In normal cells, during phagocytosis the related actin remodeling is carried out by different PI species. The PI 3, 4, 5-P3 species are produced at the phagocytic cup and initiates the endocytosis process by triggering the actin polymerization. Once internalized, the phagosome is led by PI 4, 5-P2, which is produced by the hydrolysis of PI 3, 4, 5-P3. The PI 4, 5P2 activates the ARP2/3 mediated actin polymerization via direct interaction with Rac and WASP family proteins. PI 4, 5-P2 also inhibit the proteins that enhance depolymerization or inhibition of actin polymerization (Capping protein such as gelsoline). Along with actin polymerization, PI 4, 5-P2 carries the process of phagosome maturation via enhancing the fusion of phagosome with endosomes. In $\mathrm{AD}$ condition, amyloid-beta known to down regulate the production of PI 4,5-P2 via SHIP-2 related mechanism, which inhibits the hydrolysis of PI 3,4,5-P3. The PI 3,4,5-P3 species is increasingly hydrolyzed to PI 3,4-P2, which in turn enhances the pathway of Tau phosphorylation via GSK-3 $\beta$-associated mechanism. The reduced level of PI 4, 5- P2 down regulate the actin polymerization and phagocytosis hence there is impairment in the clearance of $A \beta$ and increases in formation of phospho-Tau.

phagocytosis [26]. Cameron C Scott et al., proved the importance of phosphatidylinositol 4, 5bisphosphate (PI 4, 5-P2) in phagosome maturation process and its linkage with necessary actin remodeling. Hydrolysis of PI 4, 5-P2 from the site of phagocytosis is important for actin disassembly to proceed the phagosome maturation. The involvement of PI 4, 5-P2 in phagocytosis indicates its pivotal role in process of rapid chemotaxis and phagocytosis where rapid actin remodeling is necessary [27]. 
While other groups of R Rohatgi et al., showed the importance of N-WASP in PI 4, 5-P2 mediated actin polymerization. The PI 4, 5-P2 mediates the pathway of actin remodeling through N-WASP, cdc42, and Arp2/3 complex has been proven (Figure 1) [28].

The recent studies also suggested the importance of different PI derivatives PI (3) P and PI (4) P in the early and late stages of phagosome maturation in phagocytosis [29]. The cholesterol and sphingolipid rich membrane rafts act as a site for PI 4, 5-P2 production, and membrane-associated actin polymerization via the WASP-ARP2/3 pathway [30]. The PI 4, 5-P2 is mostly located at the inner leaflet of the membrane and regulated by lipid raft and membrane curvature. The PI 4, 5-P2 accumulates at the aggregated lipid raft regions and mediates the signaling cascade related to receptor-mediated phagocytosis [31].

In Alzheimer's disease, amyloid-beta aggregates observed to disrupts PI 4, 5-P2 metabolism (Figure 1). The oligomeric species of amyloid-beta induced decrease of PI 4, 5-P2, is depend upon the extracellular $\mathrm{Ca}^{2+}$ dyshomeostasis [15]. From the recent studies it can be stated as PI 4, 5-P2 is an important player to impose the neuronal loss and disrupted signaling cascades and hence act as a therapeutic strategy to target in $\mathrm{AD}[32]$.

\section{Phosphatidylinositol signaling in microglial migration}

Microglia is an immune cell of the brain that has surveillant nature, which is supported by high migration rates of microglia and capacity to respond to chemotaxis response [33]. The basic actin cytoskeleton is necessary to regulate the processes such as migration and surveillant nature of microglia $[4,34]$. In $\mathrm{AD}$, the accumulated abnormal proteins serve in the classical activation of microglia inducing proinflammatory response. The excessive pro-inflammatory response triggers neuroinflammation, which imparts the anti-inflammatory stage of microglia [3,4]. The plasma membrane and the underlined cortical actin network is very important for migration and phagocytosis. For the process of migration coordinated polymerization of actin filaments provides a protrusive force (lamellipodia) and thin filamentous protrusion to sense and direct the migration (filopodia) is necessary. The lamellipodia-dependent migration carried out by actin-rich protrusion lamellipodia and filopodia at leading ends. Filopodia sever as antennae of the cell which probe the environment and serve pioneer in migration [35]. Lamellipodia on the other hand produce due to coordinated actin polymerization carried by ARP2/3 complex activation [34]. The actin polymerization beneath the plasma membrane produces the protrusion that drives forward moment of cell at the leading end [36]. The membrane protrusion around the targets for phagocytosis also involved actin cytoskeleton regulation. Phosphatidylinositols induce migration by the lamellipodiadependent mechanism via inducing actin polymerization at leading ends and also provide directional clues during chemotaxis. Phosphatidylinositols regulate signaling by directly binding to actin-binding proteins and influence their activity [18]. The polarized gradient of PI 3, 4, 5-P3 after activation of chemoattractant receptor induces actin polymerization for lamellipodia-mediated migration. PI 3, 4, 5-P3 


\section{Figure 2}

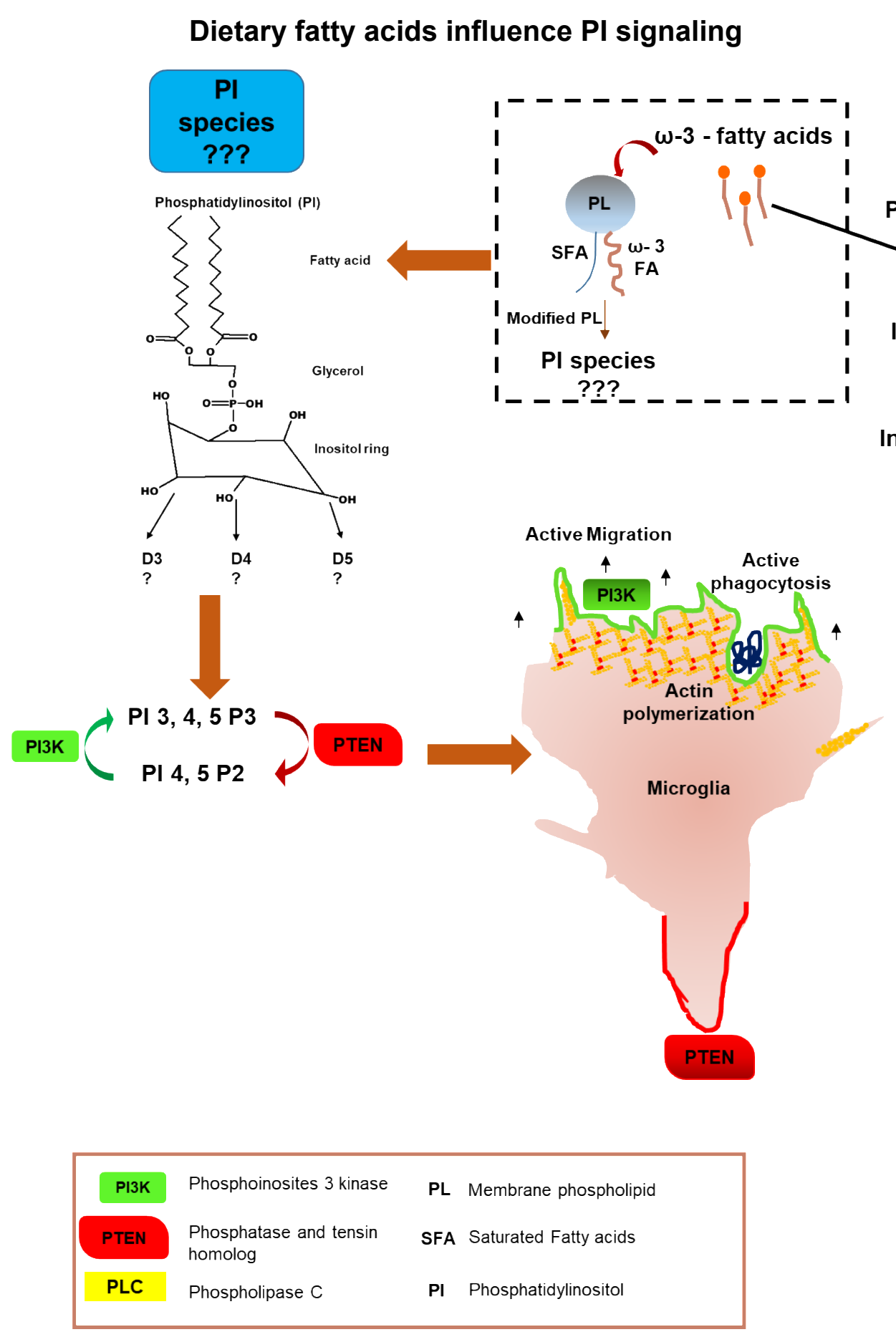

Figure 2. Dietary fatty acids influence PI signaling. The incorporation of dietary supplement of omega-3 fatty acids increases the potency to intercalate with the membrane glycerophospholipids. The increased omega-3 fatty acids in glycerophospholipids suspected to influence Phosphoinosites. Under physiological conditions fatty acids influence the type of PI species produced depending upon the phosphorylation at D3, D4, and D5 of inositol ring. 
PIP2 (PI 4, 5-P2), PIP3 (PI 3, 4, 5- P3) synthesis is maintained by the interplay of PI3K and PTEN local concentration. In highly polarized lamelliopodia bearing cell migrates with the high concentration of PIP3 at leading ends due to local concentration gradient of PI3K. The PTEN maintains directionality and retraction at rear end via inducing higher concentration of PIP2 and lower concentration of PIP3. The positive interplay between PIP3 and PIP2 would induce active phagocytosis and migration, which is supported by necessary actin polymerization in an activated cell. Polyunsaturated omega-3 fatty acids suspected to induce the phagocytosis via PI signaling. Omega-3 dietary fatty acids also inhibit PLC mediated hydrolysis of PI 4, 5-P2 into inositol 1, 4, 5 triphosphate (IP3) and diacylglycerol (DAG) which eventually initiates inflammatory response by microglia.

accumulates at the chemoattractant end and produces actin rearrangement. The gradient of PI 3, 4, 5-P3 is overproduced by carried out PI3K and hydrolysis by phosphatases and tensin homology (PTEN) at retracting ends to keep the cell on track. PI 3, 4, 5-P3 levels polarize cell by recruiting Rac1, and DOCK2 is necessary to activate ARP2/3-mediated actin filament polymerization to induce migration towards chemoattractant. The hydrolysis of PI 3, 4, 5-P3 to form a gradient towards chemoattractant produces high levels of PI 4, 5-P2 at the uropod ends, and induce actin filament assembly to address the movement of the cell. The spatial localization of PI3K and PTEN determines the membrane localization of PI 3, 4, 5P3 which creates an intracellular signaling gradient for chemotaxis [37]. The chemotactic receptors P2X, P2X4R, P2Y12R also showed activation of the PI3K pathway over stimulation by ATP/ADP and also by amyloid-beta [33]. PI derivatives hence regulate the migratory movement of cells, which is necessary to catch targets during phagocytosis (Figure 2).

\section{Dietary fatty acids govern phosphatidylinositol signaling}

Dietary fatty acids known to induce changes in cell membrane compositions and structure, which has various effects on signal transduction pathways. Dietary fatty acids influence phosphatidylethanolamine (PE), phaphatidylcholine (PC) of the cell membrane to the most and to some extent phosphatidylinositols (PI) [38]. The omega-3 fatty acids supplement of DHA and EPA specifically influence species and levels of PI in the cell, which could consider as one of the important mechanism to regulate signaling pathways and avoid cardiac arrhythmias [39]. Omega-3 fatty acids found to inhibit the phospholipase C (PLC)mediated hydrolysis of PI 4, 5-P2. The hydrolyzed product inositol 1, 4, 5-triphosphate (IP3) along with diacylglycerol (DAG) have been found to induces leukotriene (LTB4) mediated inflammatory response in neutrophils [40]. The activity of PI 3, 4, 5-P3 depends upon types of fatty acids at sn-1 and sn-2 positions of phospholipids [41]. The compositions of fatty acids at sn-1 or sn-2 positions are determined by dietary fatty acids hence dietary fatty acids could influence phosphatidylinositols pool in cell. The PUFA (Polyunsaturated fatty acid) treatment to cell significantly increases the PI species. The replaced PI species by the PUFA treatment found to inhibit tumor growth by suppressing the Akt pathway. Omega-3 
fatty acids tend to incorporate at the sn-2 position of glycerol backbone, that holds the tendency to change the species of phospholipid [41]. The acyl chain remodeling carried out by different enzymes might act as a mechanism that decides the particular PUFA chain at the sn-2 position of PI and also determines the downstream lipid signaling molecule [42]. Different fatty acids found to influence pool particular PI derivatives in the cell. Incorporation of fatty acids into cells has been found to increase particular PI derivatives. The disrupted metabolism of PI and related signaling cascade in AD could be monitored with dietary fatty acids sources.

\section{Conclusion}

Alzheimer's disease as the most common cause of dementia drags serious attention to the therapy. The two main pathological proteins are the presence of extracellular $\mathrm{A} \beta$ plaques and intracellular Neurofibrillary tangles of Tau. Apart from the pathoproteins, neuroinflammation fabricated due to microglia after aberrant activation also contributes to the disease condition and propagation. The difficulty of microglia to express anti-inflammatory response is one of the biggest challenges faced in the later stages of the disease. One of the approaches to design therapeutic strategy could be induction of the anti-inflammatory nature of microglia to overcome neuroinflammation and its side effects. Fatty acids are one of the major dietary factors, which influence microglial response. Dietary fatty acids influence antiinflammatory response by microglia is well established but it is yet to understand the important signaling molecules affected during the pathway activation. Phosphatidylinositols (PI) is a very important secondary messenger molecule that regulates various pathways like phagocytosis, migration, endocytosis, etc. PI interacts with many actin-binding proteins and other proteins through domain interaction to activate the signaling pathway. Elucidating the direct role of dietary fatty acids in activating the pathways and type of signaling molecules affected by the PI pool is a new challenge to explore. We expect that being lipid derivatives PI pool and types should show dependence on dietary fatty acids types. Since the PI levels are greatly affected in Alzheimer's disease the therapeutic strategy could be design to normalize the PI metabolism.

\section{Abbreviations}

Phosphatidylinositol- PI

Phosphatidylinositol 4, 5- bisphosphate- PI 4, 5- P2 (PIP2)

Phosphatidylinositol 3, 4, 5- triphosphate- PI 3, 4, 5- P3 (PIP3)

Phosphatidylinositol-3 kinase - PI3K

Phosphatidylinositol-5 kinase - PI5K

Protein kinase A - PKA 
Tensin homology - PTEN

Phosphatidylethanolamine - PE

Phaphatidylcholine - PC

Membrane-associated actin regulation factors-1e - Myo1e

Phospholipase C - PLC

Diacylglycerol - DAG

Alzheimer's disease- AD

Central nervous system- CNS

Amyloid- beta- $\mathrm{A} \beta$

Amyloid precursor protein- APP

Neurofibrillary tangles - NFTs

Actin-related protein complex 2/3- ARP2/3

Polyunsaturated fatty acids - PUFA

Src homology domain-containing inositol 5-phosphatase - SHIP2

\section{Declarations}

\section{Funding}

This project is supported by the in-house CSIR-National Chemical Laboratory grant MLP029526.

\section{Competing interests}

The authors declare that they have no competing interests.

\section{Ethics approval}

Not applicable

\section{Consent of participate}

Not applicable

\section{Consent of publication}

Not applicable

\section{Availability of data and materials}

Data sharing is not applicable to this article as no new data were created or analyzed in this study

\section{Author's information}

Affiliations

\section{Smita Eknath Desale}


Neurobiology Group, Division of Biochemical Sciences, CSIR-National Chemical Laboratory,

Dr. Homi Bhabha Road, 411008 Pune, India

\section{Smita Eknath Desale}

Academy of Scientific and Innovative Research (AcSIR), 411008 Pune, India

\section{Subashchandrabose Chinnathambi}

Neurobiology Group, Division of Biochemical Sciences, CSIR-National Chemical Laboratory,

Dr. Homi Bhabha Road, 411008 Pune, India

\section{Subashchandrabose Chinnathambi}

Academy of Scientific and Innovative Research (AcSIR), 411008 Pune, India

\section{Contributions}

SD and SC prepared the initial draft. SC conceived, designed, supervised, initial draft, review editing and wrote the paper. All authors read and approved the final paper.

\section{Corresponding author}

Correspondence to Subashchandrabose Chinnathambi, s.chinnathambi@ncl.res.in

\section{References}

1. Chen, G.-f., Xu, T.-h., Yan, Y., Zhou, Y.-r., Jiang, Y., Melcher, K. \& Xu, H. E. (2017) Amyloid beta: structure, biology and structure-based therapeutic development, Acta Pharmacologica Sinica. 38, 12051235.

2. Sonawane, S. K. \& Chinnathambi, S. (2018) Prion-like propagation of post-translationally modified tau in Alzheimer's disease: a hypothesis, Journal of Molecular Neuroscience. 65, 480-490.

3. Desale, S. E. \& Chinnathambi, S. (2020) Role of dietary fatty acids in microglial polarization in Alzheimer's disease, Journal of Neuroinflammation. 17, 1-14.

4. Das, R., Balmik, A. A. \& Chinnathambi, S. (2020) Phagocytosis of full-length Tau oligomers by Actinremodeling of activated microglia, Journal of Neuroinflammation. 17, 1-15.

5. Španić, E., Langer Horvat, L., Hof, P. R. \& Simic, G. (2019) Role of microglial cells in Alzheimer's disease tau propagation, Frontiers in aging neuroscience. 11, 271.

6. Pomponi, M. F., Gambassi, G., Pomponi, M. \& Masullo, C. (2010) Alzheimer's disease: fatty acids we eat may be linked to a specific protection via low-dose aspirin, Aging and disease. 1, 37.

7. Sarlus, H. \& Heneka, M. T. (2017) Microglia in Alzheimer's disease, The Journal of clinical investigation. 127, 3240-3249.

8. DiSabato, D. J., Quan, N. \& Godbout, J. P. (2016) Neuroinflammation: the devil is in the details, Journal of neurochemistry. 139, 136-153.

9. Ryan, S. D., Whitehead, S. N., Swayne, L. A., Moffat, T. C., Hou, W., Ethier, M., Bourgeois, A. J., Rashidian, J., Blanchard, A. P. \& Fraser, P. E. (2009) Amyloid- $\beta 42$ signals tau hyperphosphorylation and compromises neuronal viability by disrupting alkylacylglycerophosphocholine metabolism, Proceedings of the National Academy of Sciences. 106, 20936-20941.

10. Canevari, L., Abramov, A. Y. \& Duchen, M. R. (2004) Toxicity of amyloid $\beta$ peptide: tales of calcium, mitochondria, and oxidative stress, Neurochemical research. 29, 637-650. 
11. Kam, T.-I., Park, H., Gwon, Y., Song, S., Kim, S.-H., Moon, S. W., Jo, D.-G. \& Jung, Y.-K. (2016) FcyRIlbSHIP2 axis links $A \beta$ to tau pathology by disrupting phosphoinositide metabolism in Alzheimer's disease model, Elife. 5, e18691.

12. Chen, T. J., Wang, D. C. \& Chen, S. S. (2009) Amyloid- $\beta$ interrupts the PI3K-Akt-mTOR signaling pathway that could be involved in brain-derived neurotrophic factor-induced Arc expression in rat cortical neurons, Journal of neuroscience research. 87, 2297-2307.

13. Jimenez, S., Torres, M., Vizuete, M., Sanchez-Varo, R., Sanchez-Mejias, E., Trujillo-Estrada, L., Carmona-Cuenca, I., Caballero, C., Ruano, D. \& Gutierrez, A. (2011) Age-dependent accumulation of soluble amyloid $\beta(A \beta)$ oligomers reverses the neuroprotective effect of soluble amyloid precursor protein- $\alpha$ (SAPP $\alpha$ ) by modulating phosphatidylinositol 3-kinase (PI3K)/Akt-GSK-3 $\beta$ pathway in Alzheimer mouse model, Journal of Biological Chemistry. 286, 18414-18425.

14. Strosznajder, J. B., Zambrzycka, A., Kacprzak, M. D. \& Strosznajder, R. P. (1999) Amyloid $\beta$ peptide 25-35 modulates hydrolysis of phosphoinositides by membrane phospholipase (s) C of adult brain cortex, Journal of Molecular Neuroscience. 12, 101-109.

15. Berman, D. E., Dall'Armi, C., Voronov, S. V., McIntire, L. B. J., Zhang, H., Moore, A. Z., Staniszewski, A., Arancio, O., Kim, T.-W. \& Di Paolo, G. (2008) Oligomeric amyloid- $\beta$ peptide disrupts phosphatidylinositol-4, 5-bisphosphate metabolism, Nature neuroscience. 11, 547.

16. Gillooly, D. J., Simonsen, A. \& Stenmark, H. (2001) Phosphoinositides and phagocytosis, The Journal of cell biology. 155, 15-18.

17. Hilpelä, P., Vartiainen, M. \& Lappalainen, P. (2004) Regulation of the actin cytoskeleton by PI $(4,5)$ P 2 and PI $(3,4,5)$ P 3 in Phosphoinositides in Subcellular Targeting and Enzyme Activation pp. 117-163, Springer.

18. Saarikangas, J., Zhao, H. \& Lappalainen, P. (2010) Regulation of the actin cytoskeleton-plasma membrane interplay by phosphoinositides, Physiological reviews. 90, 259-289.

19. Deming, P. B., Campbell, S. L., Baldor, L. C. \& Howe, A. K. (2008) Protein kinase A regulates 3phosphatidylinositide dynamics during platelet-derived growth factor-induced membrane ruffling and chemotaxis, Journal of Biological Chemistry. 283, 35199-35211.

20. Doughman, R. L., Firestone, A. J., Wojtasiak, M. L., Bunce, M. W. \& Anderson, R. A. (2003) Membrane ruffling requires coordination between type l $\alpha$ phosphatidylinositol phosphate kinase and Rac signaling, Journal of biological chemistry. 278, 23036-23045.

21. Araki, N., Egami, Y., Watanabe, Y. \& Hatae, T. (2007) Phosphoinositide metabolism during membrane ruffling and macropinosome formation in EGF-stimulated A431 cells, Experimental cell research. 313, 1496-1507.

22. Zhang, Y., Cao, F., Zhou, Y., Feng, Z., Sit, B., Krendel, M. \& Yu, C.-h. (2019) Tail domains of myosin-1e regulate phosphatidylinositol signaling and $\mathrm{F}$-actin polymerization at the ventral layer of podosomes, Molecular biology of the cell. 30, 622-635.

23. Hayes, M. J., Shao, D.-M., Grieve, A., Levine, T., Bailly, M. \& Moss, S. E. (2009) Annexin A2 at the interface between F-actin and membranes enriched in phosphatidylinositol 4, 5,-bisphosphate, Biochimica et Biophysica Acta (BBA)-Molecular Cell Research. 1793, 1086-1095.

24. Janmey, P. A., Bucki, R. \& Radhakrishnan, R. (2018) Regulation of actin assembly by PI $(4,5)$ P2 and other inositol phospholipids: An update on possible mechanisms, Biochemical and biophysical research communications. 506, 307-314.

25. Tanaka, T., Tsujio, I., Nishikawa, T., Shinosaki, K., Kudo, T. \& Takeda, M. (2000) Significance of tau phosphorylation and protein kinase regulation in the pathogenesis of Alzheimer disease, Alzheimer Disease \& Associated Disorders. 14, S18-S24.

26. Botelho, R. J., Teruel, M., Dierckman, R., Anderson, R., Wells, A., York, J. D., Meyer, T. \& Grinstein, S. (2000) Localized biphasic changes in phosphatidylinositol-4, 5-bisphosphate at sites of phagocytosis, The Journal of cell biology. 151, 1353-1368. 
27. Scott, C. C., Dobson, W., Botelho, R. J., Coady-Osberg, N., Chavrier, P., Knecht, D. A., Heath, C., Stahl, P. \& Grinstein, S. (2005) Phosphatidylinositol-4, 5-bis phosphate hydrolysis directs actin remodeling during phagocytosis, The Journal of cell biology. 169, 139-149.

28. Rohatgi, R., Ho, H.-y. H. \& Kirschner, M. W. (2000) Mechanism of N-WASP activation by CDC42 and phosphatidylinositol 4, 5-bisphosphate, The Journal of cell biology. 150, 1299-1310.

29. Jeschke, A., Zehethofer, N., Lindner, B., Krupp, J., Schwudke, D., Haneburger, I., Jovic, M., Backer, J. M., Balla, T. \& Hilbi, H. (2015) Phosphatidylinositol 4-phosphate and phosphatidylinositol 3-phosphate regulate phagolysosome biogenesis, Proceedings of the National Academy of Sciences. 112, 4636-4641. 30. Rozelle, A., Machesky, L. M., Yamamoto, M., Driessens, M., Insall, R., Roth, M. G., Luby-Phelps, K., Marriott, G., Hall, A. \& Yin, H. L. (2000) Phosphatidylinositol 4, 5-bisphosphate induces actin-based movement of raft-enriched vesicles through WASP-Arp2/3, Current Biology. 10, 311-320.

31. Mu, L., Tu, Z., Miao, L., Ruan, H., Kang, N., Hei, Y., Chen, J., Wei, W., Gong, F. \& Wang, B. (2018) A phosphatidylinositol 4, 5-bisphosphate redistribution-based sensing mechanism initiates a phagocytosis programing, Nature communications. 9, 1-16.

32. Arancio, O. (2008) PIP2: a new key player in Alzheimer's disease, Cellscience. 5, 44.

33. Fan, Y., Xie, L. \& Chung, C. Y. (2017) Signaling pathways controlling microglia chemotaxis, Molecules and cells. 40, 163.

34. Desale, S. E. \& Chinnathambi, S. (2020) $\alpha$-Linoleanic acid modulates phagocytosis of extracellular Tau and induces microglial migration by actin-remodeling, bioRxiv.

35. Mattila, P. K. \& Lappalainen, P. (2008) Filopodia: molecular architecture and cellular functions, Nature reviews Molecular cell biology. 9, 446-454.

36. Le Clainche, C. \& Carlier, M.-F. (2008) Regulation of actin assembly associated with protrusion and adhesion in cell migration, Physiological reviews. 88, 489-513.

37. Castellano, E. \& Downward, J. (2010) Role of RAS in the regulation of PI 3-kinase in Phosphoinositide 3-kinase in Health and Disease pp. 143-169, Springer.

38. Williams, C. M. \& Maunder, K. (1992) Effect of dietary fatty acid composition on inositol-, cholineand ethanolamine-phospholipids of mammary tissue and erythrocytes in the rat, British journal of nutrition. 68, 183-193.

39. Nair, S. S., Leitch, J. \& Garg, M. L. (1999) Specific modifications of phosphatidylinositol and nonesterified fatty acid fractions in cultured porcine cardiomyocytes supplemented with n-3 polyunsaturated fatty acids, Lipids. 34, 697-704.

40. Sperling, R. I., Benincaso, A. I., Knoell, C. T., Larkin, J. K., Austen, K. F. \& Robinson, D. R. (1993)

Dietary omega-3 polyunsaturated fatty acids inhibit phosphoinositide formation and chemotaxis in neutrophils, The Journal of clinical investigation. 91, 651-660.

41. Gu, Z., Wu, J., Wang, S., Suburu, J., Chen, H., Thomas, M. J., Shi, L., Edwards, I. J., Berquin, I. M. \& Chen, Y. Q. (2013) Polyunsaturated fatty acids affect the localization and signaling of PIP3/AKT in prostate cancer cells, Carcinogenesis. 34, 1968-1975.

42. D'Souza, K. \& Epand, R. M. (2014) Enrichment of phosphatidylinositols with specific acyl chains, Biochimica et Biophysica Acta (BBA)-Biomembranes. 1838, 1501-1508. 